\title{
Strategies for re-opening physiotherapy OPDs post COVID-19 lockdown - A cross-sectional study
}

\author{
Gill S. Amarjot ${ }^{*}$, Saini S. Sandeep ${ }^{2}$ and Chawla S. Nistara ${ }^{3}$
}

1 Assistant Professor, College of Physiotherapy, Christian Medical College and Hospital, Brown Road, Ludhiana-141008, Punjab, India; amarjotsinghgill@gmail.com

2 Principle, Professor, College of Physiotherapy, Christian Medical College and Hospital, Brown Road, Ludhiana-141008, Punjab, India; principlecopcmcl@gmail.com

3 PG Student, Department of Physiotherapy, School of Applied Medical Sciences, Lovely Professional University, Phagwara.144411, Punjab, India.

* Correspondence: amarjotsinghgill@gmail.com; Tel.: +91 9878449971

\begin{abstract}
Background: The practices of various health-care professionals have been improvised to accommodate the on-going covid-19 pandemic situation. Different guidelines have been set in place to ease the process of re-opening of non-elective healthcare services like out-patient physiotherapy clinics. Although the measures taken should be guided by evidence based information, major consensus amongst practicing therapists needs to guide the India physiotherapy clinics.

Objective: To identify and present the opinions of different physiotherapists about the various strategies for re-opening the out-patient physiotherapy clinics.

Methods: An online cross-sectional survey was conducted. Over 169 participants were selected to participate in the survey according to the pre-decided inclusion and exclusion criteria. The data was collected and saved via google forms.

Result and conclusion: A majority of respondents had a consensus over different strategies for re-opening the physiotherapy OPDs. These were regarding different measures to be adapted including modifications in the clinic infrastructure and the practice pattern. This would help in smoothly re-instating the physiotherapy services post the covid-19 lockdown.

Keywords: COVID19, SARS CoV2, physiotherapy, healthcare system
\end{abstract}

\section{Introduction}

The COVID19 pandemic posed an unprecedented health crisis at a global level. The virus which originally began in Wuhan, China rapidly spread to numerous countries across the globe. ${ }^{[1]}$ The healthcare systems of every country affected by the virus have been tremendous pressure. The COVID19 virus or the SARS CoV2 virus is a rapidly spreading virus via aerosols.[1] The implications of the virus were initially thought to be limited to the lungs and the pulmonary system. The primary clinical symptoms include fever, cough and myalgia. ${ }^{[2]}$ However, as more and more research was carried on and data collected from various countries, it was concluded that the repercussions of the disease may be associated to almost every organ system of the body including the cardiovascular, neurological, gastrointestinal, renal and integumentary system. ${ }^{[1]}$

This pandemic has challenged the healthcare system like never before. From the exact pathophysiology to the treatment, a lot is unknown about the virus, even till date. The disease literally shows a different presentation in each and every patient that is affected by it. Antiviral agents, inflammation inhibitors/antirheumatic drugs, low molecular weight heparins, plasma, and hyperimmune immunoglobulins are some of the currently prevailing lines of treatment for COVID19. ${ }^{[3]}$ Under these circumstances, where in the world level organisations have strongly recommended physical distancing, there is a definitive need to modify healthcare practices for the safety of both, the doctors and the patients. ${ }^{[4][5]}$ The doctors that practice medicine have adapted various strategies. These include donning personal protective equipment in the out-patient facilities, phone clinics and tele-consultation, etc. ${ }^{[4]}$ 
Physiotherapy is one field of healthcare where in close contact of the patient and the therapist is almost always necessary. In the initial phase of the pandemic, many clinics had temporarily suspended their services.[6] This was because no standard safety measures for practice seemed to be in place. Various institutions have published different sets of guidelines for practice during COVID19 pandemic. ${ }^{[6]}$ Strict adherence to those guidelines is, however, difficult in the Indian physiotherapy practice scenario. This could be attributed various factors such as[7]

- lack of appropriate infrastructure to practice while maintain physical distancing,

- Unavailability of personal protective equipment suitable to allow the required degree of movement for physiotherapist, and

- Gap in training of the therapists and the staff members to practice in such a scenario.

It is a matter of utmost importance that safe standards of practice are established which are suitable for the Indian scenario. ${ }^{[6]}$ Therefore, in order to come to a consensus about the standards and modes of delivering the physiotherapy services in India during COVID-19 pandemic, we conducted this online cross-sectional survey study.

\section{Materials and Methods}

This cross sectional survey study was conducted from 20, April to 30 th May 2020, during the lockdown period. Since it would not have been feasible to collect data in-person, therefore the survey was conducted online. A google questionnaire was designed and shared with physiotherapists across various social media platforms. The questionnaire consisted of a brief introduction about the questionnaire, the background and purposes of the survey, the voluntary nature of participation and confidentiality and anonymity declaration. A set of 25 questions regarding the measures of physiotherapy practice in the COVID lockdown period were formulated. Of these 25, 18 questions were to be answered qualitatively, either by choosing from the given options or to write their own response and 7 were to be answered on a numeric rating scale from 0 to 10 .

The participants were selected according to the inclusion and exclusion criteria (Table 1). The responses from 169 participants were recorded. The results were analysed using SPSS version 26 and percentages and frequencies were calculated for the categori$\mathrm{cal} /$ nominal variables.

\begin{tabular}{|cl|l|}
\hline \multicolumn{2}{|c|}{ TABLE 1 } \\
\hline INCLISUION CRITERIA & EXCLUSION CRITERIA \\
\hline $1 . \quad \begin{array}{l}\text { Individuals running a physiotherapy clinic or } \\
\text { working in a physiotherapy clinic. }\end{array}$ & Students or non-practicing physiotherapists \\
\hline 2. & $\begin{array}{l}\text { Individuals having a minimum of under-graduate } \\
\text { degree in Physiotherapy }\end{array}$ & Unqualified practitioners \\
\hline 3. & $\begin{array}{l}\text { Individuals with a minimum of 2 years' experience } \\
\text { in clinical work. }\end{array}$ & \\
\hline 4. & Individuals having an access to a smartphone & \\
\hline
\end{tabular}




\section{Results}

A total of 169 participants completed the survey. All the participants were physiotherapists with a minimum of an under-graduate degree in physiotherapy and at least 2 years of experience in the field. According to our survey, majority of the participants, about $56.5 \%$ had a consensus to use all possible strategies for reaching out to the patients about the re-opening of the physiotherapy clinics. Social media was the second most preferred form of advertisement for about $26.1 \%$ or the participants. This can be attributed to the comfort level of the therapists with the use of social media as well as having a greater reach. One of the major dilemmas in the re-opening would be how to control the crowding the clinics. $44.9 \%$ of the participants agreed that the clinics should be fully functional with reduced staff capacity. The number of patients allowed in the clinic at a time should be limited to $2-4$ to avoid over-crowding. $60.9 \%$ agreed that only $2-4$ patients should be allowed for treatment at a point. This number would however change according to the facilities and area available in each clinic. $65.2 \%$ had a consensus on the clinical time being limited to 6 hours per day. This would help the therapists in getting ample time to ensure all the necessary sanitisation before the next clinical day.

The opinion about extent of changes in the clinical set-up varied from major to minor. These could include spacing the patient treatment area adequately to avoid patient-to-patient physical contact. Waiting areas be maintained to avoid crowding in the treatment area. Proper separation of each cabin for patients and equipment of each cabin with sanitizer dispenser is indicated. Self-organised sanitising drives need to be kept in place for the effective running of the clinics with proper safety of the patients. Physiotherapy services focus on reducing the debilitation of an individual. Therefore, the patients are often eager to continue their treatment. The same was seen in our survey. Most therapists reported that their patients were eager to come back for therapy. However, they were reluctant because of the prevailing pandemic. $73.9 \%$ of the participants suggested that the patients were eager to get back to the physiotherapy clinics, but were worried about the pandemic. Since the physiotherapy facilities generally cater to patients of all age groups, warranting visits by paediatric and geriatric population in the times of covid had to be given a thought. A major number of participants, $49.3 \%$ and $57.4 \%$ respectively, agreed that the paediatric and geriatric population should still avoid visiting the clinic with proper precautions and only if absolutely necessary.

Since the patients that belong to each of these groups exhibit lower immunity and greater susceptibility to catch infections, our survey implies that physical visits by these patients only be made with full precautions and if totally necessary. In any other circumstances, these groups should resort to online consultations and home therapy programs. ${ }^{[1]}$ The pandemic has posed an unprecedented situation to the delivery of healthcare. The changing dynamics of the pandemic makes it essential for the staff and the doctors to be updated with the recent guidelines for practice given by the WHO or the national institutions. According to our survey, $79.7 \%$ agreed that all possible means should be utilised. Self-organised dis-infection regimes with essential solutions were recommended by $84.1 \%$ of the participants for disinfecting the clinical area. $92.8 \%$ recommended that it is absolutely essential to deploy clinical staff for the screening of patients for covid-related symptoms. Regarding the most ideal method that is preferable for preparing the staff to handle clients in present situation, $23.3 \%$ suggested demonstration methods would be the best.it is of utmost importance for the staff and the doctors to keep themselves updated about the same via news, government apps and online resources. Other than these, hospital training is also indicated to apply the measures effectively. Various government advisories have suggested thermal scanning and screening for flu-like symptoms in all the patients that visit the clinic. For the same, we suggest that proper staff be deployed 
who is dedicated specifically for this screening process each day. Even the staff should be screened daily.

\section{Discussion}

To our knowledge, this would be the first online survey in India highlighting the reopening strategies for physiotherapy clinics post lock down. Since the survey was done in the online format, the participants were selectively the therapists who use or have access to a smartphone. Operating a physiotherapy facility in the post-lockdown period would not be simple. Despite various guidelines set in place by the government or the clinics at a personal level, adherence to those needs to be ensured. This is only possible if the doctors and the staff are familiar and comfortable with the new measures of practice. This can be accomplished by various initiatives such as lectures, demonstrations, mock drills and finally, hands-on practice. $46.4 \%$ agreed to use every possible way of educating and training for the same. $66.7 \%$ had a consensus on everyday screening of the staff. Training the staff is also important given that there are unlimited sources of false information that the patients or even the staff members could have access to. It is important to stop the spread of rumours so as to propagate the safety in practice. This could be done by encouraging the staff to indulge in discussions with a professional or a trusted source and avoiding the spread of rumours. $40.6 \%$ agreed that training the staff members could help in stopping the spread of rumours. $45.6 \%$ and $47.1 \%$ suggested that the patients comply totally and partially with the updated clinical guidelines respectively.

Individualised suggestions regarding the steps taken if a corona positive patient comes to your clinic and if you later on come to know that the person you attended was a corona positive case were recorded from each of the 169 participants. More than $50 \%$ strongly recommended the use of PPE, scrubs, mask, visor, gloves, and aprons/overall. The results have been represented in the Figure1. Given the new circumstances, even though the healthcare system is adopting numerous new measures, the success of the delivery of these services also depends on patient compliance. Since the methods of physical distancing and donning or personal protective equipment are not conventional, some patients could be compliant and adjusting if properly explained by the therapist. However, we do estimate that a set of population may not be fully compliant. The new measures adopted by various clinics can include all or some of the following- personal protective equipment, scrubs, mask, visor, gloves, sanitizer and apron/overalls. All the participants rated the use of all the above mentioned as highly recommended in their clinics. The covid-19 pandemic took the healthcare systems across the globe totally off-guard. But, with proper strategies formulated and implemented, healthcare professionals would be able to fight back in an effective manner.

\section{Conclusions}

The covid-19 pandemic shook the healthcare systems across the world. The delivery of healthcare services has taken a different route ever since. Physiotherapy clinics need to be re-instated in the post-lockdown phase with pandemic-friendly modifications. This process needs to be guided by scientific evidence. In the coming months, more effective strategies would be concocted.

Author Contributions: Conceptualization, G.S.A. and S.S.S.; methodology, G.S.A. and S.S.S.; software, C.S.N.; validation, G.S.A.; formal analysis, G.S.A. and S.S.S.; investigation, G.S.A.; resources, G.S.A. and S.S.S.; data curation, G.S.A. and C.S.N.; writing-original draft preparation, C.S.N.; writing - review and editing, C.S.N and G.S.A.; visualization, G.S.A.; supervision, G.S.A. and S.S.S.; project administration, G.S.A. All authors have read and agreed to the published version of the manuscript. 
Funding: This research received no external funding.

Institutional Review Board Statement: Not applicable.

Informed Consent Statement: Informed consent was obtained from all subjects involved in the study. Participation in the study was completely voluntary and anonymous. Implicit consent for the project was assumed when study participants completed the survey.

Data Availability Statement: All data generated or analyzed during this study are included in this article.

Acknowledgments: The authors extend their regards to all the respondents who participated in this survey.

Conflicts of Interest: The authors declare no conflict of interest.

\section{References}

1. Kang Y, Xu S. Comprehensive overview of COVID-19 based on current evidence. Dermatol Ther. 2020;33(5):e13525. doi:10.1111/dth.13525

2. Umakanthan S, Sahu P, Ranade AV, et al. Origin, transmission, diagnosis and management of coronavirus disease 2019 (COVID-19). Postgrad Med J. 2020;96(1142):753-758. doi:10.1136/postgradmedj-2020-138234

3. Stasi C, Fallani S, Voller F, Silvestri C. Treatment for COVID-19: An overview. Eur J Pharmacol. 2020;889:173644. doi:10.1016/j.ejphar.2020.173644

4. Iyengar K, Mabrouk A, Jain VK, Venkatesan A, Vaishya R. Learning opportunities from COVID-19 and future effects on health care system. Diabetes Metab Syndr. 2020;14(5):943-946. doi:10.1016/j.dsx.2020.06.036

5. Lakhani A, Sharma E, Gupta K, Kapila S, Gupta S. Corona Virus (COVID-19) and its Impact on Health Care Workers. J Assoc Physicians India. 2020;68(9):66-69.

6. Greenfield B, Musolino GM. Technology in rehabilitation: ethical and curricular implications for physical therapist education. Journal of Physical Therapy Education. 2012;26(2):81-90

7. Haines KJ, Berney S. Physiotherapists during COVID-19: usual business, in unusual times. J Physiother. 2020;66(2):67-69. doi:10.1016/j.jphys.2020.03.012

8. American Physical Therapy Association. The Vision Statement. Available at: http://www.apta.org/Vision. Accessed April 3, 2020

9. Information and resources about COVID-19. World Confederation for Physical Therapy website. https://www.wcpt.org/news/Novel-Coronavirus-2019-nCoV. Updated April 9, 2020. Accessed April 13, 2020.

10. Khalid A, Ali S. COVID-19 and its Challenges for the Healthcare System in Pakistan [published online ahead of print, 2020 Aug 13]. Asian Bioeth Rev. 2020;1-14. doi:10.1007/s41649-020-00139-x

11. The Topol Review. Preparing the healthcare workforce to deliver the digital future. NHS Health Education England. Available at: https://topol.hee.nhs.uk. Accessed April 19, 2020 